\title{
Gene expression profiling and its use in adenocarcinomas of unknown primary origin: A case report
}

\author{
ANA CEBOLLERO DE MIGUEL, ROBERTO PAZO CID, JAVIER MARTINEZ TRUFERO, \\ ISABEL PAJARES BERNAD, LOURDES CALERA URQUIZU, \\ JORGE HERNANDO CUBERO and ANTONIO ANTON TORRES
}

Department of Medical Oncology, Miguel Servet University Hospital, Zaragoza 50009, Spain

Received October 21, 2014; Accepted July 10, 2015

DOI: $10.3892 / 01.2015 .3572$

\begin{abstract}
Carcinomas of unknown primary origin account for $3-5 \%$ of all malignancies. The current literature suggests that metastatic dissemination is able to occur in the absence of primary tumor growth. In metastatic disease that is difficult to diagnose, the origin usually remains unknown even after an exhaustive evaluation of immunohistochemistry (IHC) markers. In the current study, a 49-year-old male presented with lymph nodes metastases of unknown origin. The excisional biopsy of an inguinal node revealed an adenocarcinoma growth pattern, but the IHC could not determine the primary origin. A gene profiling test was performed to complete the diagnosis and a salivary gland adenocarcinoma was diagnosed with $90 \%$ probability. Subsequently, the patient underwent appropriate chemotherapy for salivary gland adenocarcinoma, and exhibited an improved partial response. The present case study highlights the importance of an accurate diagnosis of the primary tumor and the use of all the current tools available in order to provide patients with the best treatment possible.
\end{abstract}

\section{Introduction}

Cancers of unknown primary (CUPs) represent a heterogeneous group of metastatic tumors for which the site of origin cannot be diagnosed on a standardized diagnostic work-up at the time of diagnosis. CUP accounts for 3-5\% of all malignancies $(1,2)$.

At present, CUP is the fifth and eighth most common cancer diagnosis in women and men, respectively (3). The majority of cases have an unfavourable prognosis, with a life expectancy that ranges from 6 to 9 months (4).

Correspondence to: Dr Ana Cebollero de Miguel, Department of Medical Oncology, Miguel Servet University Hospital, 1-3 Isabel la Catolica Avenue, Zaragoza 50009, Spain

E-mail: draernes_1983@hotmail.com

Key words: cancer of unknown primary, adenocarcinoma, gene expression profiling, salivary gland cancers
Without a specific diagnosis, the management of these patients is more difficult, as the treatment is typically empirical, with non-specific antineoplastic drugs used in combination in a range of chemotherapy regimens, but with no one treatment schedule superior to the others (5).

Primary tumor cells exhibit characteristic genetic expression that is maintained among the metastatic cells; therefore, it is possible to identify the primary site by comparing the genetic profile of the metastatic cells to a genetic profile database of different types of tumors. Therefore, various gene expression profiling methods have been developed in order to aid in identifying the primary tumor (6).

The current study presents the case of a patient with a diagnosis of CUP in which gene expression profiling was performed and the patient benefited from a specific systemic chemotherapy treatment, established as a result of having taken into account this information of the presumed primary site. Written informed consent was obtained from the patient.

\section{Case report}

A 49-year-old male, non-smoker, with no notable medical history, was admitted to the Miguel Servet University Hospital (Zaragoza, Spain) in September 2013 due to right inguinal pain and swelling of the ipsilateral leg. The patient had a good performance status of 1 and a normal physical examination, with the exception of the swelling of the right leg. Inguinal ultrasonography (US) did not reveal any pathology. Since no symptom improvement was achieved over time, US was performed again in February 2014, which revealed multiple enlarged abdominal lymph nodes, including retroperitoneal and para-aortic nodes, the largest measuring $32 \mathrm{~mm}$ in diameter.

Total body computed tomography (CT) and fluorodeoxyglucose positron emission CT were performed and a poliadenopathic condition, involving the left axillary, cervical, supraclavicular, mediastinal, retroperitoneal, para-aortic, iliac and bilateral inguinal lymph nodes, was found. The largest node, in the right inguinal area, measured $41 \mathrm{~mm}$ at its largest diameter.

Laboratory tests revealed a markedly increased carcinoembryonic antigen (CEA) level (33 U/ml; normal range for non-smokers, $0-2.5 \mathrm{U} / \mathrm{ml}$; normal range for 
Table I. Immunohistochemistry results.

Result $\quad$ Antigen

Positivity

Negativity
CK7, CK8, CAM5.2, CEA, EMA, AE1:AE3, E-cadherin, GDFP-15

CD45, S-100, vimentin, PLAP, inhibin, CD30, CK20, CK5/6, PSA, TTF1, CDX2, RE, RP, HER2

CK, cytokeratin; CAM5.2, anti-cytokeratin 5.2; CEA, carcinoembryonic antigen; EMA, epithelial membrane antigen; AE, anti-pan cytokeratin; GDFP-15, gross cystic disease fluid protein-15; PLAP, anti-placental alkaline phosphatase antibody; CD, cluster of differentiation; PSA, prostate-specific antigen; TTF1, thyroid transcription factor-1; CDX2, caudal-type homeobox protein; RE, estrogen receptor; RP, progesterone receptor; HER2, human epidermal growth factor-2.
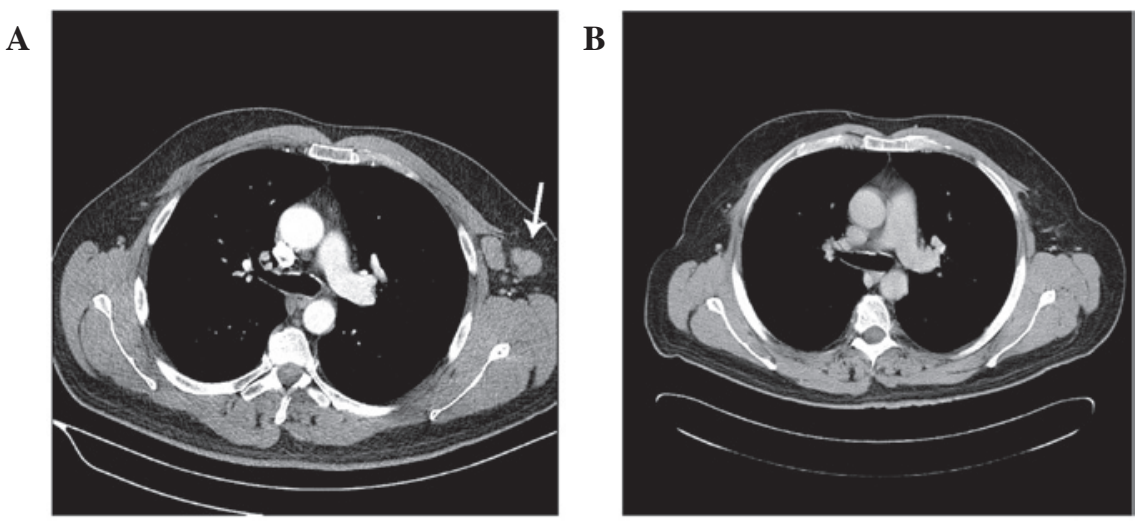

Figure 1. Left axillary lymph-node (A) prior to and (B) following chemotherapy.

A

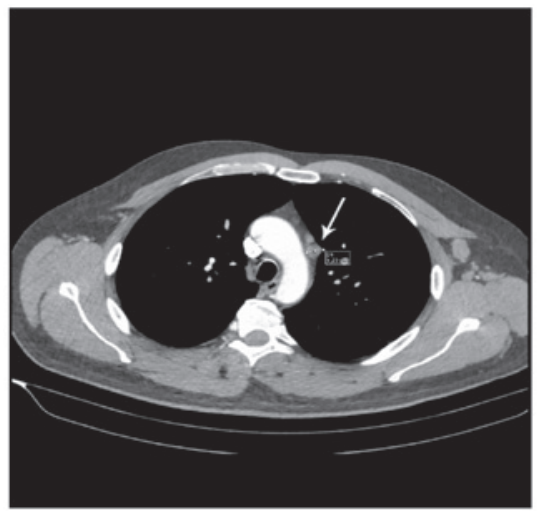

B

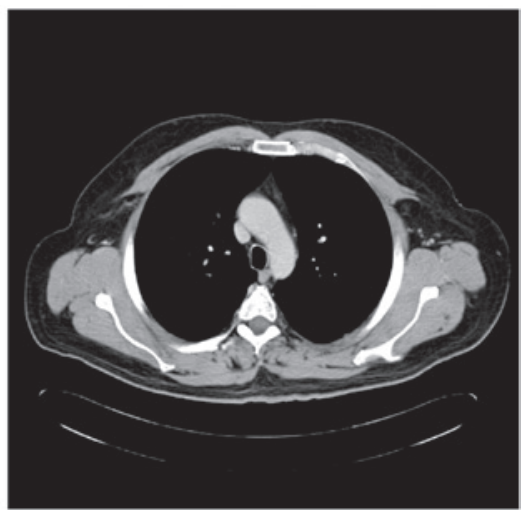

Figure 2. Mediastinal lymph-node (A) prior to and (B) following chemotherapy.

smokers, 0-5 U/ml), but the $\alpha$-fetoprotein, $\beta$-human chorionic gonadotropin, prostate-specific antigen (PSA), cancer antigen 19.9 and $\beta 2$ microglobulin levels were within laboratory normal ranges. On March 3, 2014, the patient underwent an excisional biopsy of the right inguinal lymph node. The pathology report showed an adenocarcinoma, without identifying the primary site. An extensive range of immunohistochemistry (IHC) tests were performed, which resulted in positivity for cytokeratin (CK)7, CK8, CAM5.2, CEA, epithelial membrane antigen (EMA), AE1:AE3, E-cadherin and GDFP-15, and negativity for ALC, S-100, vimentin, PLAP, inhibin, cluster of differentiation 30, CK20, CK5/6, PSA, thyroid transcription factor-1, CDX-2, RE, RP and human epidermal growth factor-2 (Table I). No EGFR gene mutation was found. The Ki-67 proliferation index was $70 \%$.
This IHC profile suggested the lungs, breasts, salivary glands and sweat glands as the most likely primary tumor locations. In order to select the most adequate therapy, a tumor gene expression profiling test was performed. This novel diagnostic technique allows the prediction of the tumor origin based on gene expression patterns that are retained from the tissues of origin (7). A salivary gland adenocarcinoma was the main diagnosis with a probability of $90 \%$. Breast adenocarcinoma could not be excluded, with a probability of $6 \%$. The patient received standard advanced salivary gland first-line chemotherapy consisting of a $50 \mathrm{mg} / \mathrm{m}^{2}$ cisplatin, $50 \mathrm{mg} / \mathrm{m}^{2}$ doxorubicin and $500 \mathrm{mg} / \mathrm{m}^{2}$ cyclofosfamide (CAP) regimen every three weeks.

After 6 cycles of CAP for 18 weeks, CT revealed a partial response of all the lymph nodes, with a significant decrease 

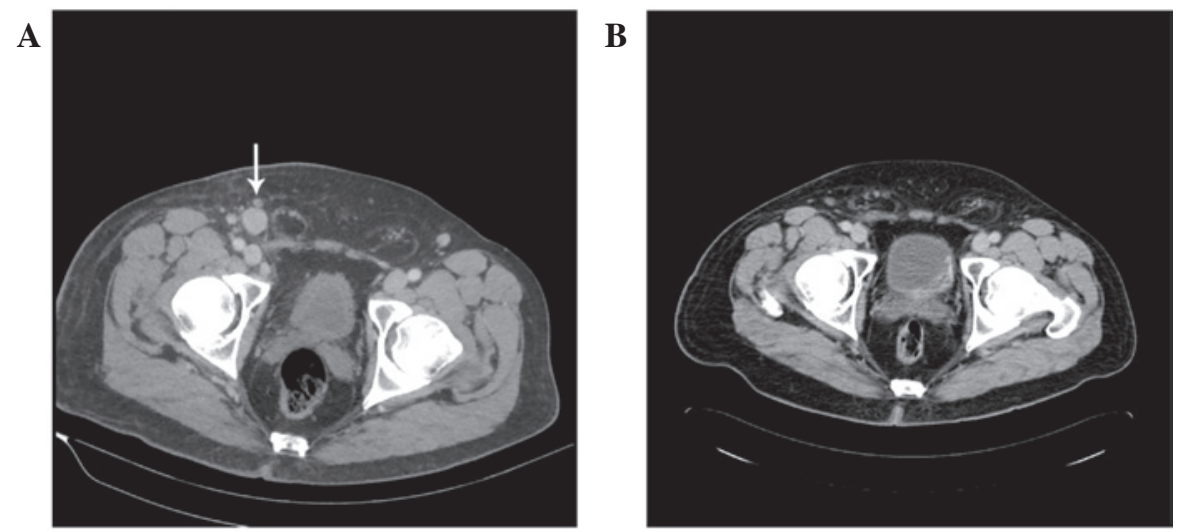

Figure 3. Right inguinal lymph-node (A) prior to and (B) following chemotherapy.

in size of the left axillary, mediastinal and right inguinal lymph nodes (Figs. 1-3). Subsequently, the patient continued follow-up with stable disease until Februrary 2015, when progression occurred (brain and liver metastases). Two different regimens of chemotherapy (carboplatin-taxol and vinorelbine) were administered with no response. In July 2015, the patient presented a worsened performance status and was admitted due to seizures.

\section{Discussion}

By definition, CUP is a histologically confirmed malignant tumor that is not compatible with a primary neoplasm in the biopsy area, and which has an origin that remains unclear once a thorough clinical history, complete physical examination and basic complementary studies have been performed (8).

CUP can be classified as an adenocarcinoma (50-70\%), poorly-differentiated carcinoma (20-30\%), squamous cell carcinoma (5-8\%) or undifferentiated malignant neoplasm (2-3\%) depending on its histological characteristics $(9,10)$.

There is little published data on adenocarcinomas of unknown origin with lymphadenopathy presentation. The majority of the studies published describe mediastinal and cervical lymph node presentations (11-14). Different approaches have been used for treatment; in one approach, surgery is the cornerstone of the treatment, whereas in another, platinum-based chemotherapy is used, with progression-free survival times of 38 and 30 months, respectively $(11,14)$.

Recent advances made with regard to specific treatments for each cancer type together with the continuous development of novel therapies should benefit the primary tumor prediction in patients with CUP, providing improved therapies and prognosis.

Traditionally, the majority of patients have been treated with empiric chemotherapy, including platinum- and taxane-based chemotherapy regimes, with poor results (median survival time, 6-8 months) $(15,16)$. As randomised controlled trials have not established any effective therapies for such patients, empirical systemic treatments that consider the performance status of the patients are widely accepted. In recent years, novel targeted drugs and personalized medicine have had increasing roles in oncological therapy. As a result, with more specific and targeted drugs, therapy with a single empiric combination chemotherapy regimen becomes increasingly inadequate.

Gene expression profiling assays correctly identified the site of origin in $85 \%$ of cases in blinded studies of tumors of known origin, and was found to favorably compare with IHC staining $(17,18)$. The best use of gene expression profiling to form a diagnosis appears to be for cases with inconclusive IHC staining. The site-specific treatment of carcinomas of unknown primary site based on gene expression profiling and/or IHC predictions appears to result in improvements in the overall patient outcome $(19,20)$. Therefore, gene expression profiling should be included in the diagnosis of a patient when IHC staining is not able to predict a single site of origin. Site-specific treatment, based on a tissue of origin diagnosis, should replace empiric chemotherapy in patients with CUP.

Salivary gland cancer (SGC), such as that diagnosed in the present case, is uncommon and accounts for $<5 \%$ of all cancers of the head and neck (21). Different types of SGC have considerably variable histological patterns and behavior. The tumors are classified according to the 2005 World Health system (22), in which 24 histological subtypes are listed. In total, $>75 \%$ of all SGCs are accounted for by mucoepidermoid carcinoma, adenoid cystic carcinoma and adenocarcinoma, as described by the Memorial Sloan-Kettering experience (23). The characteristics of slow growth, multiple local recurrences and prolonged clinical course, often with delayed distant metastasis, have all been attributed to SGCs (23).

Salivary gland malignancies may exhibit distant metastatic disease, with the best example data shown in studies of adenoid cystic carcinoma. The most common site of distant metastasis is the lungs (24), followed by the liver and bones.

Metastatic presentation of unknown primary is uncommon for salivary gland carcinomas. Florentine et al (25) reported a patient who had metastatic bone disease from unknown primary as the initial diagnosis, and the patient's further medical history showed a record of early-stage adenoid cystic carcinoma (ACC) of the soft palate, which had been diagnosed and resected 3 months earlier. The bone histopathology was compatible with ACC.

To the best of our knowledge, the present study is the first report of multiple lymph nodes metastases as the only presentation in salivary gland adenocarcinoma. 
CAP, the most commonly studied regimen for cancer, is an actively used regimen for SGC (26-28). In a study by Alberts et al (29) responses were observed that included 2 patient who experienced complete remission, from a total of 5 patients. Kaplan et al (26) recorded 5 objective responses, including 1 complete response, from a total of 6 patients. Additionally, 3 complete and 3 partial remissions, from a total of 13 patients, were reported in the study by Dreyfuss et al (27).

The present patient benefited from a site-specific treatment; however, if the primary site had not been known, a different regimen of chemotherapy would have had been used and since no optimum chemotherapy regimen has been identified for CUP, a worse patient outcome would be expected (30). The patient underwent gene expression profiling with CancerTYPE ID (BioTheranostics, Inc., San Diego, CA, USA), in order to aid in the identification of the primary site. The CancerTYPE ID assay is a 92 -gene reverse transcription-polymerase chain reaction assay that allows the identification of 30 main tumor types and 54 subtypes (17). There is also another gene expression profiling assay currently commercially available, which uses 64 tissue-specific microRNAs to enable the identification of 42 tumor types, using a microarray technology known as the Cancer Origin Test (Rosetta Genomics, Rehovot, Israel) (18). These two assays were validated using biopsies of tumors from known primary sites. Biopsies were taken from either the primary site or a metastatic site; tumors with well-differentiated and poorly-differentiated histology were included. In these validation studies, each of which included specimens from several hundred tumors, the two assays each correctly identified $85 \%$ of the tumors included $(17,18)$.

Regarding the present case study, the patient benefited from a site-specific treatment according to the prediction of the gene expression profiling assay, and experienced a partial response for all lesions following use of a chemotherapy widely used in salivary gland adenocarcinoma. Gene expression profiling is a valuable addition to a diagnostic evaluation. Nevertheless, ongoing clinical trials are required to refine these recommendations.

\section{References}

1. Pavlidis $\mathrm{N}$ and Fizazi K: Cancer of unknown primary (CUP). Crit Rev Oncol Hematol 54: 243-250, 2005.

2. Varadhachary GR, Abbruzzese JL and Lenzi R: Diagnostic strategies for unknown primary cancer. Cancer 100: 1776-1785, 2004.

3. Altman E and Cadman E: An analysis of 1539 patients with cancer of unknown primary site. Cancer 57: 120-124, 1986.

4. Pavdilis N, Briasoulis E, Hainsworth J and Greco FA: Diagnostic and therapeutic management of cancer of an unknown primary. Eur J Cancer 30: 1990-2005, 2003.

5. Golfinopoulos V, Pentheroudakis G, Salanti G, Nearchou AD, Ioannidis JP and Pavlidis N: Comparative survival with diverse chemotherapy regimens for cancer of unknown primary site: Multiple-treatments meta-analysis. Cancer Treat Rev 35: 570-573, 2009

6. Varadhachary GR, Talantov D, Raber MN, Meng C, Hess KR, Jatkoe T, Lenzi R, Spigel DR, Wang Y, Greco FA, et al: Molecular profiling of carcinoma of unknown primary and correlation with clinical evaluation. J Clin Oncol 26: 4442-4448, 2008.

7. Su AI, Welsh JB, Sapinoso LM, Kern SG, Dimitrov P, Lapp H, Schultz PG, Powell SM, Moskaluk CA, Frierson HF Jr and Hampton GM: Molecular classification of human carcinoma by use of gene expression signatures. Cancer Res 61: 7388-7393, 2001.
8. Greco FA and Hainsworth JD: Cancer of unknown primary site. In: Cancer: Principles \& Practice of Oncology. DeVita VT Jr, Hellman S and Rosenberg SA (eds). 6th edition. Lippincott Williams \& Wilkins, Philadelphia, pp2537-2560, 2001.

9. Van de Wouw AJ, Janssen-Heijnen ML, Coebergh JW and Hillen HF: Epidemiology of unknown primary tumours; incidence and population-based survival of 1285 patients in Southeast Netherlands, 1984-1992. Eur J Cancer 38: 409-413, 2002.

10. Hess KR, Abbruzzese MC, Lenzi R, Raber MN and Abbruzzese JL: Classification and regression tree analysis of 1000 consecutive patients with unknown primary carcinoma. Clin Cancer Res 5: 3403-3410, 1999.

11. Harada H, Yamashita Y, Kuraoka K and Taniyama K: Sequential mediastinal lymphadenectomy of an unknown primary tumor. The Ann Thorac Surg 95: 687-689, 2013.

12. Nakano $T$, Endo $S$, Endo $T$, Hasegawa $T$, Nakayama $M$, Sugiyama Y and Hironaka M: Multimodal treatment for multistation mediastinal lymph node adenocarcinoma: A case report. Ann Thorac Cardiovasc Surg 18: 136-139, 2012.

13. Sorgho-Lougue LC, Luciani A, Kobeiter H, Zelek L, Malhaire C, Deux JF, Brun B, Piedbois P and Rahmouni A: Adenocarcinomas of unknown primary (ACUP) of the mediastinum mimicking lymphoma: CT findings at diagnosis and follow-up. Eur J Radiol 59: 42-48, 2006.

14. Pepper C, Pai I, Hay A, Deery A, Wilson P, Williamson P and Pitkin L: Investigation strategy in the management of metastatic adenocarcinoma of unknown primary presenting as cervical lymphadenopathy. Acta Otolaryngol 134: 838-842, 2014.

15. Briasoulis E, Kalofonos H, Bafaloukos D, Samantas E, Fountzilas G, Xiros N, Skarlos D, Christodoulou C, Kosmidis P and Pavlidis N: Carboplatin plus paclitaxel in unknown primary carcinoma: A phase II Hellenic cooperative oncology group study. J Clin Oncol 18: 3101-3107, 2000.

16. Culine S, Lortholary A, Voigt JJ, Bugat R, Théodore C, Priou F, Kaminsky MC, Lesimple T, Pivot X, Coudert B, et al: Cisplatin in combination with either gemcitabine or irinotecan in carcinomas of unknown primary site: Results of a randomized phase II study-trial for the French study group on carcinomas of unknown primary (GEFCAPI 01). J Clin Oncol 21: 3479-3482, 2003.

17. Erlander MG, Ma XJ, Kesty NC, Bao L, Salunga R and Schnabel CA: Performance and clinical evaluation of the 92-gene real-time PCR assay for tumor classification. J Mol Diagn 13: 493-503, 2011.

18. Meiri E, Mueller WC, Rosenwald S, Zepeniuk M, Klinke E, Edmonston TB, Werner M, Lass U, Barshack I, Feinmesser M, et al: A second-generation microRNA-based assay for diagnosing tumor tissue origin. Oncologist 17: 801-812, 2012.

19. Greco FA, Lennington WJ, Spigel DR, Varadhachary GR and Hainsworth JD: Carcinoma of unknown primary site: Outcomes in patients with a colorectal molecular profile treated with site-specific chemotherapy. J Cancer Ther 3: $37-43,2012$

20. Hainsworth JD, Schnabel CA, ErlanderMG, Erlander MG, Haines DW III and Greco FA: A retrospective study of treatment outcomes in patients with carcinoma of unknown primary site and a colorectal cancer molecular profile. Clin Colorectal Cancer 11: 112-118, 2012.

21. Laurie SA and Licitra L: Systemic therapy in the palliative management of advanced salivary gland cancers. J Clin Oncol 24: 2673-2678, 2006.

22. Barnes L, Eveson JW, Reichart P and Sidransky D (eds): Tumours of the Salivary Glands. In: World Health Organization Classification of Tumors: Pathology and Genetics of Head and Neck Tumours. World Health Organization, Lyon, France, pp209-282, 2005.

23. Spiro RH: Salivary neoplasms: Overview of a 35-year experience with 2,807 patients. Head Neck Surg 8: 177-184, 1986.

24. Hamper K, Lazar F, Dietel M, Caselitz J, Berger J, Arps H, Falkmer U, Auer G and Seifert G: Prognostic factors for adenoid cystic carcinoma of the head and neck: A retrospective evaluation of 96 cases. J Oral Pathol Med 19: 101-107, 1990.

25. Florentine BD, Fink T, Avidan S, Braslavsky D, Raza A and Cobb CJ: Extra-salivary gland presentations of adenoid cystic carcinoma: A report of three cases. Diagn Cytopathol 34: 491-494, 2006.

26. Kaplan MJ, Johns ME and Cantrell RW: Chemotherapy for salivary gland cancer. Otolaryngol Head Neck Surg 95: $165-170,1986$ 
27. Dreyfuss AI, Clark JR, Fallon BG, Posner MR, Norris CM Jr and Miller D: Cyclophosphamide, doxorubicin and cisplatin combination chemotherapy for advanced carcinomas of salivary gland origin. Cancer 60: 2869-2872, 1987.

28. Licitra L, Cavina R, Grandi C, Palma SD, Guzzo M, Demicheli R and Molinari R: Cisplatin, doxorubicin and cyclophosphamide in advanced salivary gland carcinoma: A phase II trial of 22 patients. Ann Oncol 7: 640-642, 1996.

29. Alberts DS, Manning MR, Coulthared SW, Koopmann CF Jr and Herman TS: Adriamycin/cis-platinum/cyclophosphamide combination chemotherapy for advanced carcinoma of the parotid gland. Cancer 47: 645-648, 1981.
30. Golfinopoulos V, Pentheroudakis G, Salanti G, Nearchou AD, Ioannidis JP and Pavlidis N: Comparative survival with diverse chemotherapy regimens for cancer of unknown primary site: Multiple-treatments meta-analysis. Cancer Treat Rev 35: 570-573, 2009. 